Research Article

\title{
Temporin A and Bombinin H2 Antimicrobial Peptides Exhibit Selective Cytotoxicity to Lung Cancer Cells
}

\author{
Lucy Swithenbank (D), ${ }^{1}$ Phillipa Cox, ${ }^{1}$ Llinos G. Harris $\mathbb{D}^{1},{ }^{1}$ Edward Dudley, \\ Kathryn Sinclair, ${ }^{1}$ Paul Lewis, ${ }^{1}$ Floriana Cappiello, ${ }^{2}$ and Claire Morgan ${ }^{1}{ }^{1}$ \\ ${ }^{1}$ Swansea University Medical School, Institute of Life Science, Swansea University, Swansea SA2 8PP, UK \\ ${ }^{2}$ Department of Biochemical Sciences "A. Rossi Fanelli", Sapienza University of Rome, Rome, Italy \\ Correspondence should be addressed to Claire Morgan; c.morgan@swansea.ac.uk
}

Received 9 April 2020; Revised 21 May 2020; Accepted 30 May 2020; Published 29 June 2020

Academic Editor: Osman Kucuk

Copyright (c) 2020 Lucy Swithenbank et al. This is an open access article distributed under the Creative Commons Attribution License, which permits unrestricted use, distribution, and reproduction in any medium, provided the original work is properly cited.

Background. Recently, antimicrobial peptides (AMPs) have been investigated for their use in cancer therapy. They have been reported to selectively target and kill cancer cells whilst leaving normal healthy cells unaffected. Certain Anura AMPs have expressed selective cytotoxicity against tumour cells. Aim. To test the potential of Anura AMPs bombinin H2, bombinin H4, temporin A, and temporin L for use as therapeutic agents for non-small cell lung carcinoma (NSCLC). Methods. Cytotoxic effects on NSCLC cell lines A549 and Calu-3 and normal epithelial cell line Beas-2B were tested using the CellTox Green Cytotoxicity Assay. Their haemolytic effects on human erythrocytes were also tested for their clinical relevance. Cell membrane profiling, using MALDI-TOF, was performed to ascertain if membrane characteristics of the NSCLC and Beas-2B cell lines may contribute to the AMPs mode of action. Results. Bombinin H4 $(100-1.5 \mu \mathrm{M}, p<0.05)$ and temporin A $(100-50 \mu \mathrm{M}, p<0.05)$ showed selective cytotoxicity towards the NSCLC cell lines. Furthermore, they exhibited low levels of haemolytic activity (bombinin H4, $0.061 \%$; temporin A, $0.874 \%$ ) comparable to untreated cells. Cell membrane profiling showed the phospholipid composition of normal epithelial cell line Beas-2B to be divergent from the cancerous cell lines. However, there was an overlap in the phospholipid profiles of the NSCLC cell lines supporting the hypothesis that the AMPs may have a selective affinity via the membrane composition of cancerous cell lines. Conclusion. These results suggest that bombinin H4 and temporin A show potential for application in lung cancer therapies. Further in vitro and in vivo studies are required to develop a greater understanding of their use as anticancer agents.

\section{Introduction}

In 2018, it was reported that there were an estimated 17 million new cancer cases and 9.6 million cancer related deaths worldwide, with lung cancer being the most common cause of death [1]. Lung cancer is commonly treated via chemotherapy [2]. However, the success of this treatment is limited due to the rise of chemotherapeutic resistance in cancer cells due to alterations occurring within membrane transporter proteins such as multidrug resistance (MDR) proteins and P-glycoprotein. Furthermore, the nonspecific targeting of the chemotherapy agents results in their toxicity towards healthy dividing cells, causing deleterious side effects such as nausea, diarrhoea, hair loss, anaemia, and infections, all of which reduce the patients' overall quality of life. Unfortunately, the prognosis of lung cancer patients has not shown the same level of improvement over recent years as has been observed in other cancers such as breast and prostate [3]. Following an average of 4-6 months' posttreatment initiation, patients suffer disease progression, highlighting the long term ineffectiveness of the current treatments and aggressiveness of the disease [4]. The 5-year survival rate for non-small cell lung cancer (NSCLC) is currently only $10-20 \%$ [5]. It is therefore imperative that new drug treatments that have neither the toxicity nor the mechanisms of resistance associated with conventional chemotherapy are developed. 
Antimicrobial peptides (AMPs) are a group of compounds that are a conserved element of the innate immune response [6], found in all species investigated such as bacteria, fungi, plants, insects, birds, fish, amphibians, and mammals [6,7]; these small peptides are between 12 and 50 amino acids long [8]. They have been shown to effectively kill a wide range of micro-organisms such as viruses, fungi, and both Gram-positive and -negative bacteria [9]. AMPs are grouped into two sets: those which are toxic to bacteria and cancer cells but not to noncancerous mammalian cells and those which are toxic towards all three cell types [10]. AMPs specific selectivity for cancer cells over normal epithelial cells is generally attributed to several complimenting characteristics between the cancer cell membranes and the AMPs. Such characteristics include their ability to interact electrostatically with each other, hydrophobicity of the AMPs represented as therapeutic index, and the sequence of the AMP. The electrostatic interaction between the AMP and the target cell membrane is one of the key drivers of interaction, with AMPs typically being cationic with a net positive charge between +2 and +7 due to an excess of basic amino acids such as arginine, lysine, and histidine [11]. Not all AMPs possess anticancer properties but those that do, anticancer peptides (ACPs), have been recognised as being more specific in their effects, producing less harmful side effects than chemotherapy and traditional therapies $[10,12,13]$.

AMPs found in Anura frog skin secretions have been used medicinally for many years. The dorsal skin secretions collected from Anura are amongst the richest sources of naturally formed AMPs, acting as part of the defence mechanisms to protect the frog skin from pathogen invasion and predator ingestion [14]. The Anura expels the AMPs, along with other molecules such as alkaloids, neuropeptides, and biogenic amines, in large amounts in response to threat or injury [15]. Each Anura species secretes a unique set of AMPs which leads to the variety in properties such as antimicrobial, anticancer, and haemolytic effects [16]. Certain Anura AMPs have been observed to express selective cytotoxicity against tumour cells, such as AMPs from African claw frogs from the Pipidae family which have demonstrated tumouricidal properties against small cell lung cancer cell lines [17] and bladder cancer cell lines [18] as well as a range of hematopoietic cell lines [19].

The purpose of this study is to investigate antimicrobial peptides from the Anura species Rana temporaria (temporin A and temporin L) [20] and Bombina variegata (bombinin $\mathrm{H} 2$ and bombinin $\mathrm{H} 4$ ) [21] to determine whether they display selective cytotoxicity towards lung cancer cells, thus offering potential as new chemotherapeutic agents to treat lung cancer. The percentage of cell viability with AMPs at varying concentrations and time points was tested using lung cancer cell lines (A549 and Calu-3) as well as noncancer cell line (Beas-2B). Furthermore, cell membrane characterisation was analysed to determine if membrane composition may affect the AMPs mode of action.

\section{Materials and Methods}

2.1. Peptides. Temporin A [FLPLIGRVLSGIL], temporin L [FVQWFSKFLGRIL], bombinin H2 [IIGPVLGLVGSALGGLLKKI], and bombinin $\mathrm{H} 4$ [I-(D-allo-1) GPVLGLVGSALGGLLKKI] were synthesized by Selleck Chemicals (Houston, TX, USA).

2.2. Peptide Validation. Matrix assisted laser desorption/ ionisation (MALDI) mass spectrometry analysis was carried out on the samples following the addition of $100 \mu \mathrm{l}$ of $0.1 \%$ formic acid to solubilise the peptide. The matrix solution was made up of $\alpha$-cyano-4-hydroxycinnamic acid $(10 \mathrm{mg} / \mathrm{ml})$ combined with a $50: 50$ solution acetonitrile and $0.1 \%$ trifluoroacetic acid. When all the samples were dry, the MALDI plate was loaded into the Voyager-DE STR Biospectrometry Workstation (Applied Biosystems, Framingham, MA). Data acquisition parameters were set: ion mode, positive; instrument mode, reflector; instrument range, 1000 to $4000 \mathrm{Da}$; low mass gate, $1000 \mathrm{Da}$; total scans, 100 shots per spectrum; accelerating voltage, $20,000 \mathrm{~V}$. The results were analysed using Voltage Data Explorer ${ }^{\mathrm{TM}}{ }^{\text {version }} 4.0$ (Applied Biosystems, Framingham, MA). The spectrum was calibrated against the reference proteins (angiotensin, bradykinin, and neurotensin).

Electrospray ionisation (ESI) mass spectroscopy was used in combination with MALDI mass spectroscopy to further validate the peptide identities. Each peptide was mixed with $100 \mu \mathrm{l}$ of methanol before being pipetted into the LTQ Orbitrap XL (Thermo Fisher Scientific, Waltham, MA). The peptides were fragmented in higher-energy collisional dissociation (HCD) mode with an isotope band width of $1.5 \mathrm{~m} / \mathrm{z}$.

2.3. Antimicrobial Properties. Staphylococcus epidermidis 1457 and S. epidermidis 5179-R1 were cultured on horse blood agar plates for 24 hours at $37^{\circ} \mathrm{C}$ and subsequently used to inoculate $3 \mathrm{ml}$ of prewarmed Tryptic Soy Broth (TSB; Becton Dickinson, Cockeysville, USA). Precultures were grown in a shaking incubator for $2-3$ hours at $37^{\circ} \mathrm{C}$ and used to inoculate $200 \mu \mathrm{l}$ fresh TSB in 96-well tissue culture plates (NUNC, Thermo Fisher Scientific, Roskilde, Denmark) to a starting $\mathrm{OD}_{600}$ of 0.05 . To each test well, $100 \mu \mathrm{M}$ of peptides was added. This concentration was chosen as it was the upper limit of the concentrations being investigated for anticancer potential. Untreated bacteria were used as controls.

The 96-well plate was inserted into a BMG FLUOstar Omega microplate reader (BMG LABTECH, Germany) set to a wavelength of $600 \mathrm{~nm}$. The OD of each well was read every hour over 24-hour period, to determine the effect of the AMPs on the growth rate/population density of the bacteria. All experiments were performed in triplicate, and each strain and condition tested in triplicate.

2.4. Cell Culture. NSCLC cell line A549 (ATCC-CCL185) and normal epithelial cell line Beas-2B (ATCC CRL-9609) 
were routinely grown in DMEM (Corning, Thermo Fisher Scientific, Basingstoke, UK) supplemented with $10 \%$ foetal bovine serum (FBS) (South American Origin, Biosera, Uckfield, UK), 2 mM L-glutamine (Gibco, Thermo Fisher Scientific, Basingstoke, UK), and 1\% penicillin/streptomycin (Gibco, Thermo Fisher scientific, Basingstoke, UK). The NSCLC cell line Calu-3 (ATCC HTB55) was routinely grown in RPMI-1640 (Corning, Thermo Fisher Scientific, Basingstoke, UK) supplemented with $10 \%$ FBS, $2 \mathrm{mM}$ L-glutamine, and $1 \%$ penicillin/streptomycin. All cells were incubated at $37^{\circ} \mathrm{C}$ and $5 \% \mathrm{CO}_{2}$.

\subsection{Cytotoxicity Assays}

2.5.1. CellTox Green Cytotoxicity Assay. The CellTox Green Cytotoxicity Assay was used to determine reduction in membrane integrity due to AMP treatment. CellTox Green Dye was mixed with cell stock made in phenol-free, serumfree medium to a ratio of $1 \mu \mathrm{l}$ dye: $1000 \mu \mathrm{l}$ cell stock. Phenolfree medium decreases the background noise of the fluorescence reading so that a more accurate reading can be taken. This solution was then used to dilute the peptide to specific concentrations $(100 \mu \mathrm{M}, 50 \mu \mathrm{M}, 25 \mu \mathrm{M}, 12.5 \mu \mathrm{M}$, $6.25 \mu \mathrm{M}, 3 \mu \mathrm{M}, 1.5 \mu \mathrm{M})$. Once the cells were dosed, the plates were maintained at a temperature of $37^{\circ} \mathrm{C}$ and $5 \% \mathrm{CO}_{2}$. Untreated cells, comprising media and CellTox Green Dye, were used as a control; cell lysis buffer (provided in the CellTox Cytotoxicity Assay kit) was used as a positive control, indicating cell death.

Fluorescence readings were taken using a POLARstar Omega plate reader (BMG LABTECH, Germany) at 24 hours in accordance with published literature $[22,23]$. The plate was read from the bottom optic at $485-510 \mathrm{~nm}$ excitation and 520-530 $\mathrm{nm}$ emission, with gain adjusted to 1500 OD. The gain was optimised so that the maximum value was not reached by any of the peptides for any cell lines over the $24 \mathrm{~h}$ treatment. Readings were corrected against the blank average. Only the peptides which were cytotoxic to the NSCLC cell lines, A549 and Calu-3, were tested for selectivity on the normal lung epithelial cell line, Beas-2B. All experiments were performed in triplicate.

2.5.2. Haemolytic Assay. The HaemoScan Biomaterial Haemolytic Assay (HaemoScan, Netherlands) was used to investigate the cytotoxicity of the AMPs on human erythrocytes. Only the AMPs which displayed selective cytotoxicity towards the cell lines A549 and Calu-3 were tested for haemolytic properties.

The protocol of the assay was followed according to the manufacturers' instructions. In summary, the erythrocyte suspension was prepared by adding $5 \mathrm{ml}$ of the supplied wash buffer to the erythrocyte suspension and centrifuging at $400 \times \mathrm{g}$ for 10 minutes. The supernatant was then removed. This step was repeated twice with the wash buffer, and the steps were then repeated replacing wash buffer with dilution buffer twice more. The final pellet was then suspended in $5 \mathrm{ml}$ of dilution buffer to form a colourless supernatant.
The minimum concentration of each peptide that had a significant effect on A549 and/or Calu-3 without significantly effecting noncancer cells (Beas-2B) was added to $0.5 \mathrm{ml}$ of erythrocyte suspension (bombinin $\mathrm{H} 4,6.25 \mu \mathrm{M}$; temporin $\mathrm{A}, 50 \mu \mathrm{M})$. A haemoglobin calibration curve was also produced to establish the haemoglobin concentrations present in the treatment groups of the erythrocyte suspension after exposure to the AMPs.

Both the calibrator and treatment groups were then incubated at $37^{\circ} \mathrm{C}$ for 24 hours. After 24 hours of incubation the samples were centrifuged at $4000 \times \mathrm{g}$ for 1 minute and $20 \mu \mathrm{l}$ of the supernatant pipetted into a 96-well plate along with $180 \mu \mathrm{l}$ of assay buffer. The plate was agitated on a plate shaker before being read on a BMG FLUOstar Omega microplate reader (BMG LABTECH, Germany). The absorbance was read at three wavelengths, 380,415, and $450 \mathrm{~nm}$ (Harboe method), and the calculation $(2 \times 415)-(450+380)$ was applied to give a final OD.

Haemoglobin concentrations of the test samples were then established by comparing the treatment readings against the haemoglobin calibration curve. The haemoglobin concentration of the treatment groups was presented as a percentage of the total haemoglobin present in the sample, expressed as percentage haemolysis. All experiments were performed in triplicate.

\subsection{Cell Membrane Characterisation}

2.6.1. Phospholipid Extraction and MALDI-TOF MS Analysis. Prior to phospholipid analysis, cultured cell lines were harvested as follows: culture medium was discarded from the flasks; the cells were washed with $10 \mathrm{ml}$ PBS and were subsequently incubated with $3 \mathrm{ml} 0.025 \%$ trypsin/ EDTA for 3-5 min. The reaction was terminated by the addition of $10 \mathrm{ml}$ cell culture medium, and the samples were centrifuged at $10,000 \times \mathrm{g}$ for $5 \mathrm{~min}$.

Cell pellets were then solubilised in $200 \mathrm{ul}$ of chloroform: methanol $(2: 1)$ and then diluted $(1: 5)$ in either dihydroxybenzoic acid (DHB) at a concentration of $10 \mathrm{mg} /$ $\mathrm{ml}$ in methanol containing $0.1 \%$ trifluoroacetic acid or para-nitroaniline (PNA) at a concentration of $10 \mathrm{mg} / \mathrm{ml}$ in chloroform: methanol $(2: 1)$. One microliter of each mixture was then spotted on a clean MALDI plate (Applied Biosystems). Samples were analysed using a Voyager-DE STR, MALDI-TOF mass spectrometer (Applied Biosystems, Framingham, MA). A $337 \mathrm{~nm}$ specific laser wavelength was used to ensure that only the outer membrane was detected. Data acquisition parameters were set: time delay of 200 nanoseconds, total scans of 100 shots per spectrum, and accelerating voltage of $20,000 \mathrm{~V}$. The two matrices (DHB and PNA) were utilised and run in both the positive and negative modes, respectively. The $\mathrm{m} / \mathrm{z}$ signal was aligned across each sample within 0.5 of each other using Excel. The results were analysed and normalised using Voltage Data Explorer ${ }^{\mathrm{TM}}$ version 4.0 (Applied Biosystems, Framingham, MA). Any background noise was removed, $\mathrm{m} / \mathrm{z}$ values between 1 and 1600 were collected, and new spectra were established. 
2.6.2. Identification and Charge of Phospholipids. Possible identities of each membrane component at specific $\mathrm{m} / \mathrm{z}$ values were assigned according to the work of Estrada and Yappert [24], e.g., PC (40:6), which represents the headgroup (number of carbons in the fatty acid chain: number of double bonds).

2.7. Statistical Analysis. Intragroup data readings were checked for outliers using the Grubbs test, identifying outliers with a $p$ value greater than 0.05 when compared with the expected distribution of results. Any outliers identified were excluded from further statistical analysis. A one-sample Kolmogorov-Smirnov (K-S) test was carried out on each data group to test the normality of the data to determine appropriate further data analysis tests. All data was shown to be of a non-normal distribution and therefore a Kruskal-Wallis nonparametric analysis was carried out. To identify any significant differences between groups, a Mann-Whitney test was used to test individual treatment groups against the untreated control group. A $p$ value of $<0.05$ was considered significant.

\section{Results}

MALDI and ESI mass spectrometry confirmed that the synthetically made identities of each peptide accurately represented the peptide found from the natural source, Anura bombinin $\mathrm{H} 2$ and its diastereomer bombinin $\mathrm{H} 4$, containing a D-alloisoleucine at the second N-terminal residue as a result of a posttranslational modification, temporin A, and temporin L [25].

3.1. Antimicrobial Properties. Untreated S. epidermidis 1457 and 5179-R1 underwent rapid proliferation throughout the initial 8 hours, reaching a maximum $\mathrm{OD}_{600}$ of 0.95 and 0.7 , respectively, after 10 hours before entering a stationary phase. For S. epidermidis 1457, bombinin H2, bombinin H4, and temporin L $(100 \mu \mathrm{M})$ significantly inhibited proliferation over the 24 hours $(p \leq 0.001$; Figure 1$)$, whilst only bombinin $\mathrm{H} 2$ and bombinin $\mathrm{H} 4(100 \mu \mathrm{M})$ significantly inhibited growth of S. epidermidis 5179-R1 ( $p \leq 0.001$; Figure $1(\mathrm{~b}))$.

3.2. Cell Cytotoxicity. When bombinin $\mathrm{H} 2$ was applied to the A549 cell line, statistical analysis showed significant cell death between the untreated cells and the treated cells at concentrations ranging from $12.5 \mu \mathrm{M}$ to $50 \mu \mathrm{M}(p \leq 0.05)$. For Calu-3 cells, bombinin $\mathrm{H} 2$ resulted in significant cell death, when compared to the control, at concentrations of $50 \mu \mathrm{M}$ and $100 \mu \mathrm{M} \quad(p \leq 0.001)$ (Figure 2(a)). However, significant cell death also occurred when the noncancerous cell line Beas-2B was treated with bombinin $\mathrm{H} 2$ at concentrations of $12.5 \mu \mathrm{M}$ to $100 \mu \mathrm{M}$. These data show that bombinin $\mathrm{H} 2$ is not selectively cytotoxic to cancer cells.

Bombinin H4 proved to be highly toxic to the A549 cell line after 24-hour exposure, with significant cell death being observed from $1.5 \mu \mathrm{M}$ to $100 \mu \mathrm{M}(p \leq 0.05)$. However, in the Calu-3 cell line, significant cell death was only observed in the higher concentrations, $50 \mu \mathrm{M}$ and $100 \mu \mathrm{M}(p \leq 0.001)$, when compared to the untreated cells (Figure 2(b)). In the Beas-2B cell line, significant cell death was observed at $12.5 \mu \mathrm{M}-100 \mu \mathrm{M}(p \leq 0.05)$. As bombinin H4 showed selective cytotoxicity to A549 cells at the lowest concentration tested but not in the Beas-2B cells, it is suggested that bombinin $\mathrm{H} 4$ exhibits some level of selective cytotoxicity.

Interestingly, temporin A showed a dual effect on the A549 cell line. At concentrations of $50 \mu \mathrm{M}$ and $100 \mu \mathrm{M}$ $(p \leq 0.001)$, significant cell death was observed. However, at concentrations of $3 \mu \mathrm{M}-25 \mu \mathrm{M}$, a significant increase in cell viability was observed $(p \leq 0.050)$. For Calu-3 cells, $50 \mu \mathrm{M}$ and $100 \mu \mathrm{M}$ temporin $\mathrm{A}$ induced significant cell death $(p \leq 0.01)$ when compared to the untreated cells but, unlike A549, did not increase cell viability at the lower concentrations (Figure 2(c)). When temporin A was applied to the Beas-2B cell lines, significant cell death was only observed at $100 \mu \mathrm{M}(p \leq 0.05)$. From these results, it could be suggested that temporin $\mathrm{A}$ is selectively cytotoxic at $50 \mu \mathrm{M}$.

The A549 cell line treated with temporin L at concentrations of $1.5 \mu \mathrm{M}-25 \mu \mathrm{M}(p \leq 0.05)$ resulted in significant cell death when compared to the untreated cells. In the Calu3 cell line, temporin $\mathrm{L}$ induced significant cell death across the whole range of concentrations, $1.5 \mu \mathrm{M}-100 \mu \mathrm{M}$ $(p \leq 0.05)$, when compared to the untreated cells (Figure 2(d)). As with Calu-3 cell line, Beas-2B cell line showed significant cell death across the whole temporin $\mathrm{L}$ concentration range, $1.5 \mu \mathrm{M}-100 \mu \mathrm{M} \quad(p \leq 0.05)$, when compared to the untreated cells. Temporin L, therefore, was shown not to be selectively cytotoxic.

3.3. Haemolytic Properties. In the previous cytotoxicity investigations, only bombinin $\mathrm{H} 4$ and temporin $\mathrm{A}$ were identified to have selective cytotoxicity potential towards lung cancer cells, A549, and/or Calu-3 cell types, over noncancerous cell line Beas-2B. Therefore, due to their selective cytotoxic characteristics, only bombinin $\mathrm{H} 4$ and temporin A were investigated for haemolytic activity after $24 \mathrm{~h}$ treatment at $6.25 \mu \mathrm{M}$ and $50 \mu \mathrm{M}$, respectively. Interestingly, whilst bombinin $\mathrm{H} 4$ did not produce a cytotoxic effect on erythrocytes, levels of haemolysis were significantly less than untreated cells $(p \leq 0.001)$, suggesting that bombinin H4 may have a protective effect on erythrocytes viability. Temporin A, though nontoxic to erythrocytes with a haemolysis value comparable to untreated cells $(p \leq 1.000)$, did not display the same protective attributes as bombinin H4 (Table 1).

3.4. Cell Membrane Characterisation. The phospholipid profile of each cell line membrane was studied in order to understand the possible differences it presents to an AMP and hence the potential impact of membrane structure on AMP cytotoxic activity. Principle component analysis (PCA) using positive ionisation MALDI-TOF (Figure 3(a)) showed that Beas-2B, A549, and Calu-3 cell lines were distinct from one another whilst staying clustered within their cell type, indicating that membrane phospholipid expression was different among all three cell lines. Negative-mode MALDITOF principle component analysis showed the phospholipid 


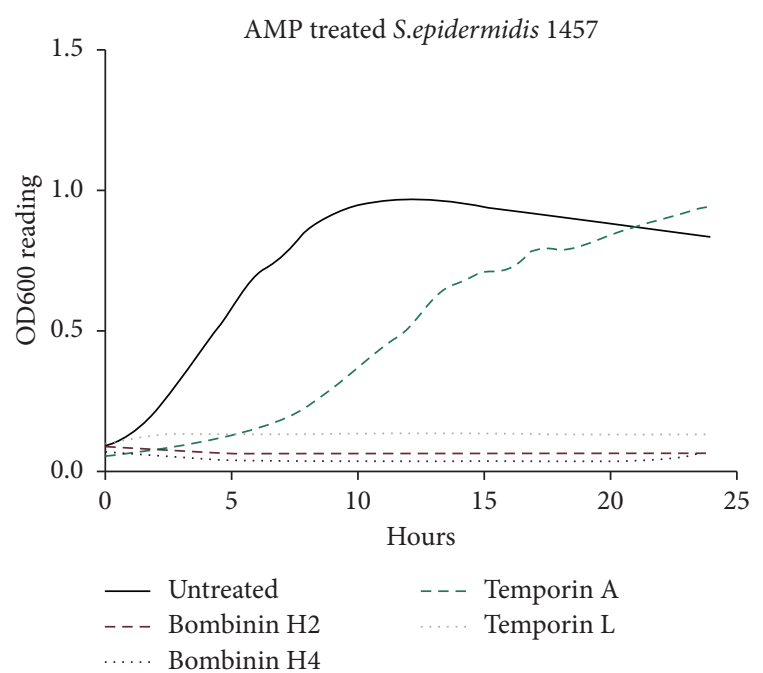

(a)

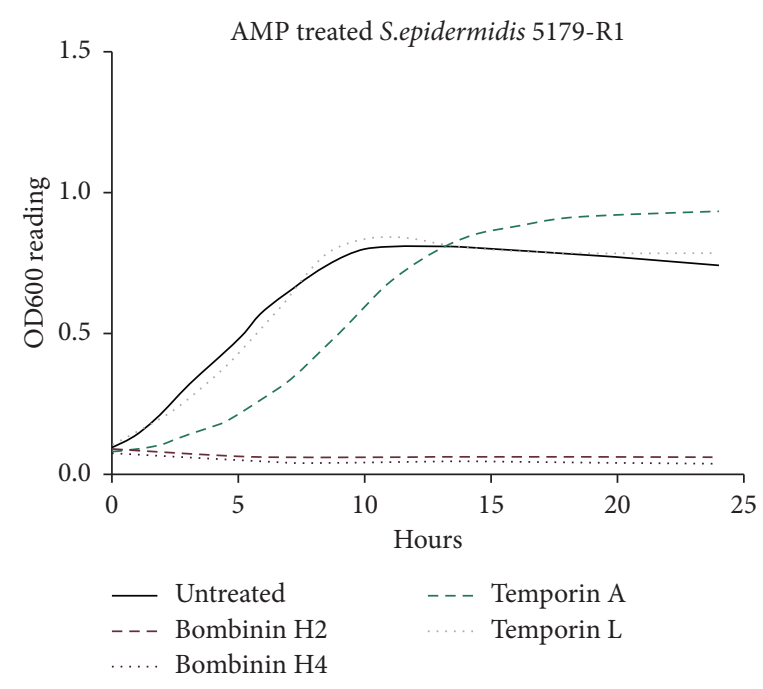

(b)

Figure 1: (a) Effects of the AMPs on Staphylococcus epidermidis 1457 over a 24-hour treatment period. (b) Effects of the AMPs on Staphylococcus epidermidis 5179-R1.

composition of normal epithelial cell line Beas-2B to be divergent from the cancerous cell lines. However, we found that there was an overlap of the phospholipid profiles of the A549 and Calu-3 cancer cell lines (Figure 3(b)) which may help explain why the AMPs had a similar effect upon the cancerous cell lines but reacted differently with the noncancerous Beas-2B.

Membrane phospholipid expression profiles were also compared to try and identify any differences in cell membrane composition, which may account for the different effect the AMPs had on cell toxicity. When comparing the normal epithelial cell line Beas-2B with the NSCLC cell line A549, twenty-four significant peaks were acquired where the phospholipid was more abundant in either Beas-2B or A549 $(p<0.05)$; thirteen of the twenty-four had a $p$ value of $<0.005$ (Figure 3(b)). When comparing Beas-2B cell line with NSCLC cell line Calu-3, thirty-two membrane components were found to be significantly different $(p<0.05)$ with twenty of those having a $p$ value of 0.005 .

The variation in abundance of the phospholipids between cell lines (Figure 4) represents how the presence affects the overall cell membrane charge. The average relative intensities (\%) of the identified lipid species were allocated their corresponding charge, the abundance of which was used to create a percentage of negative and positive phospholipids within each cell line. Beas-2B cells had a greater percentage of positive phospholipids and fewer negative phospholipids in comparison to A549 and Calu-3 cell lines $(p<0.05)$. When Calu-3 and A549 cells were compared, Calu-3, the stage III cancer cell line, was found to be more negatively charged $(1.7 \%)$ than the A549 cell line.

\section{Discussion}

Cancer treatments can vary: surgery, chemotherapy, radiotherapy, or a combination of these treatments. With specific regard to lung cancer, $90 \%$ of individuals with small cell lung cancers will initially respond to chemotherapeutic treatment, but almost all will relapse with multidrug resistant disease, whilst patients with non-small cell lung cancer have a low response rate to chemotherapy due to inherent drug resistance [26]. In recent years, AMPs have attracted attention as potential anticancer drugs due to reports that they can selectively target and kill cancer cells whilst leaving normal healthy cells unaffected, thus making them attractive candidates for investigation. AMPs found in Anura have been used for medicinal purposes for years, yet only a handful of studies have reported the cytotoxic effects of Anura AMPs against different cancer types [17, 18]; therefore, we sought to determine if the AMPs bombinin $\mathrm{H} 2$, $\mathrm{H} 4$ (from the Anura species Bombina variegate) and temporin A and L (from the Anura species Rana temporaria) displayed selective anticancer activity, thus offering potential as new chemotherapeutic agents to treat lung cancer.

In this study we have been able to demonstrate that temporin A and bombinin H4 interacted more effectively towards the NSCLC cell lines than in the normal lung cell line. Temporin A showed the greatest potential as an anticancer agent due to its ability to initiate significant cell death in both NSCLC cell lines, at the same concentration, without significant cell death in the Beas-2B cell line. Interestingly, bombinin H4 selectively killed A549 cells but not Calu-3 cells. The difference in the ability of bombinin $\mathrm{H} 4$ to induce cytotoxic activity in the cancer cell lines suggests a difference in the cell membrane protein composition of the two cancer cell lines as a possible explanation for this observed effect. The membrane phospholipid profile demonstrated that the cell lines clustered into distinct groups, suggesting that the cells could have a distinct phospholipid compositional signature on their outer leaflets. However, upon investigation of the outer membranes in negative-mode MALDITOF, PCA showed that whilst Beas-2B cells have a distinct phospholipid signature from that of the cancerous cells, the cancerous cells overlapped, implying that the cancer cell 


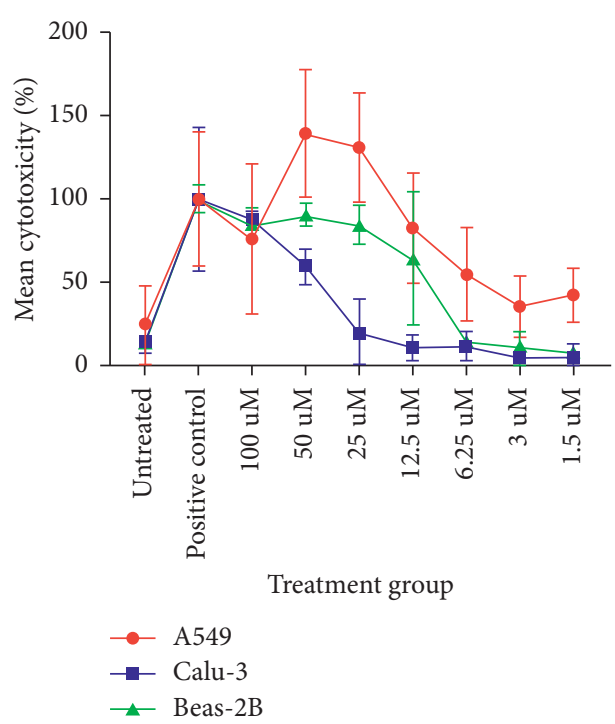

(a)

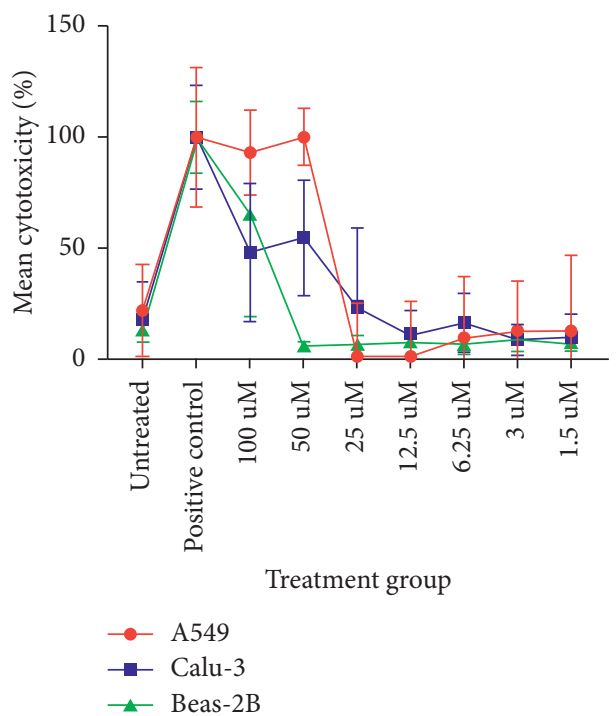

(c)

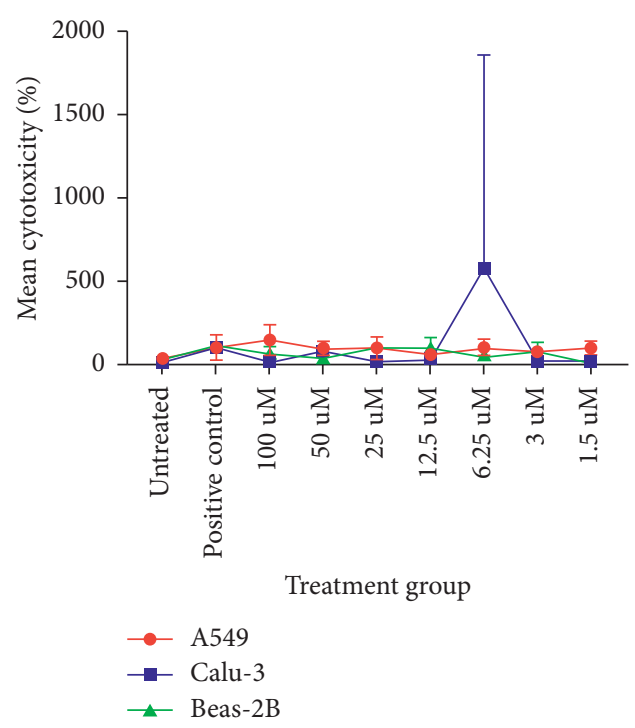

(b)

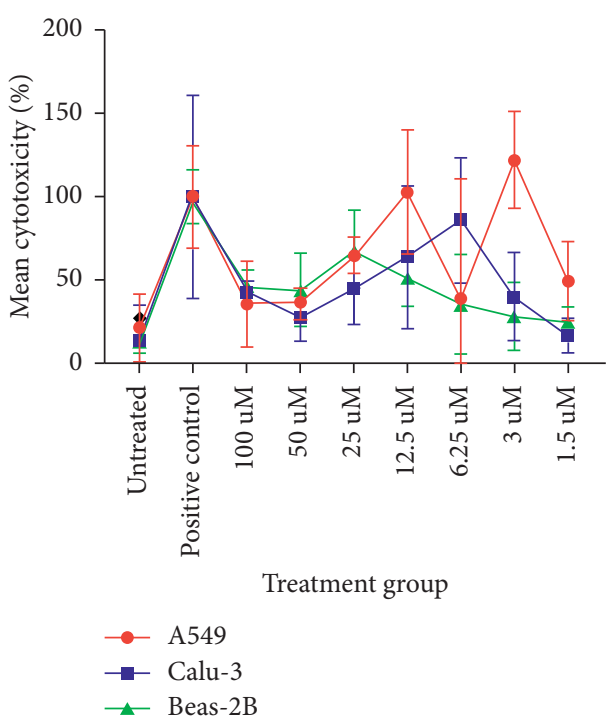

(d)

Figure 2: The cytotoxic effects observed on A549, Calu-3, and Beas- $2 \mathrm{~B}$ cell lines induced by (a) bombinin $\mathrm{H} 2$, IC50 value: $5.632 \times 10^{-7}$ ( $95 \%$ CI $3.935 \times 10^{-7}$ to $\left.7.9 \times 10^{-7}\right)$; (b) bombinin H4, IC50 value: $5.637 \times 10^{-7}\left(95 \%\right.$ CI $3.935 \times 10^{-7}$ to $\left.7.9 \times 10^{-7}\right)$; (c) temporin A, IC50 value: $5.637 \times 10^{-7}\left(95 \%\right.$ CI $3.935 \times 10^{-7}$ to $\left.7.9 \times 10^{-7}\right)$; and (d) temporin L, IC50 value: $5.632 \times 10^{-7}\left(95 \%\right.$ CI $3.935 \times 10^{-7}$ to $\left.7.9 \times 10^{-7}\right)$.

TABle 1: The mean percentage haemolysis induced by AMPS bombinin $\mathrm{H} 4$ and temporin A.

\begin{tabular}{lc}
\hline Treatment group & Mean percentage haemolysis (\%) \\
\hline Untreated cells & 0.8252 \\
Total haemolysis & 100 \\
Bombinin H4 & 0.0606 \\
Temporin A & 0.8739 \\
\hline
\end{tabular}

lines have similar phospholipid profiles. Thus, cell membrane phospholipid composition, alone, cannot explain why bombinin H4 was cytotoxic to A549 cells but not Calu-3.

The charge of the cell membrane can also affect the affinity of the AMP for selective targeting of cancerous cells. As expected, we found that normal epithelial Beas-2B cells had a greater percentage of positive phospholipids and fewer negative phospholipids in comparison to the A549 and Calu3 cell lines. Although PCA could not identify specific differences between the cell lines, normal mammalian cells predominantly contain zwitterionic phospholipids within their outer leaflet such as PC and SM, whilst negatively charged phospholipids such as PS and PG reside on the inner leaflet. In contrast, in cancer the negatively charged phospholipids become externalised to the outer leaflet [27-30], enhancing electrostatic interaction between the AMPs and cancer cells.

When A549 and Calu-3 phospholipid chargers were compared, we found Calu-3, the stage III cancer cell line, to be more negatively charged (1.7\%) than the A549 cell line. Given that AMPs exert their cytotoxic effects through 


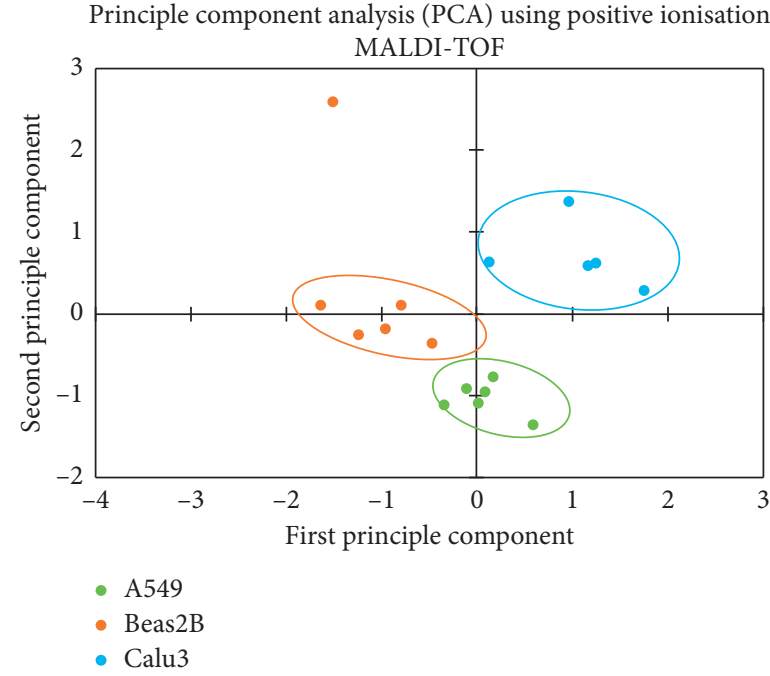

(a)

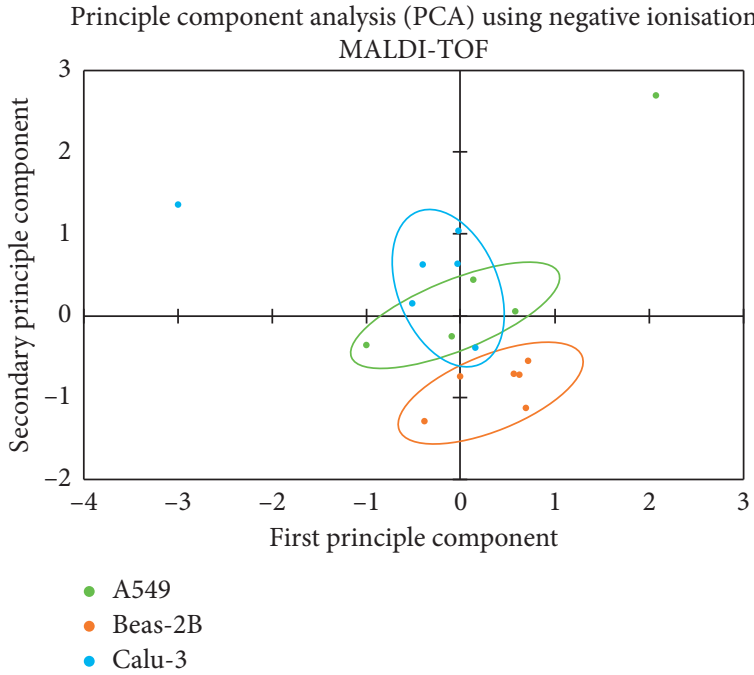

(b)

FIgURE 3: (a) PCA scatterplot of normalised MALDI-TOF DHB derived data. Phospholipid spread for six repeats of three in vitro cell lines is shown (A549, Beas-2B, Calu-3). The phospholipid profiles of each cell line appear to be clearly distinct from one another. (b) The relationship between six repeats for three in vitro cell lines via PCA is shown. There is an overlap in the phospholipid expression between he cancerous cell lines, whereas the normal cell line was separate from the cancerous cells.

electrostatic interaction, it is surprising that the more negatively charged Calu-3 cells were not as susceptible to bombinin $\mathrm{H} 4$ as the A549 cells.

Thus, another factor that needs to be taken into consideration is the properties of the AMPs themselves. Temporin A was cytotoxic to both cancer cell lines at the same concentration, yet bombinin $\mathrm{H} 4$ induced cell death at much lower concentrations in A549 than Calu-3. Thus, it may be argued that the peptide sequence of the AMP, itself, plays a major role in its cytotoxic potential. Indeed, the sequence of an AMP can affect its ability to interact with cell membranes. Several early studies have demonstrated that peptide helicity is important for toxicity [31, 32]. Incorporation of D-amino acids into cell lytic peptides causes a reduction in their cytotoxicity on mammalian cells $[33,34]$. Bombinin $\mathrm{H} 4$ has single L-D-isomerization, which may be enough to reduce the peptides alpha-helical content, in turn reducing its ability to bind to cell membranes. The disruption of the alpha-helical content can lead to the disruption of the hydrophobic face of the AMP with the positively charged residue on the cell membrane [35]. This in turn could prevent the binding of the AMP to the membrane from weakened polar attraction. This structural change, in combination with a slightly lower positive charge on the Calu- 3 membrane, may help explain why bombinin $\mathrm{H} 4$ was not as cytotoxic to this cell line.

Many studies investigating the anticancer properties of AMPs use cell lines from different cancer types [12, 36, 37]; our study highlights the importance of investigating several cancer cell lines, especially from the same cancer type, to determine if any observed AMP anticancer effect is consistently observed across multiple cell lines or is cell line specific.

For AMPs to be viable anticancer agents they must not induce haemolytic responses in the patient. Temporin A and bombinin $\mathrm{H} 4$ were tested on human erythrocytes to investigate their haemolytic properties at the minimum concentrations required to cause significant cell death in NSCLC cell lines, A549 and Calu-3. Encouragingly, for temporin $\mathrm{A}$, we observed a haemolysis value comparable to untreated cells. With bombinin $\mathrm{H}$, haemolysis was significantly less than the untreated control cells, suggesting that it actually had a protective effective on erythrocyte viability. The membrane of erythrocytes is asymmetrically distributed with glycolipids, phosphatidylcholine, and sphingomyelin expressed on the outer membrane to form a hydrophilic environment. The surface charge of erythrocytes has been shown to have a zeta potential of $10 \mathrm{mV}$, which is considered to be a neutral charge [38]. Thus, the neutral charge of the erythrocytes may not attract the predominantly positively charged AMPs. Furthermore, the relative size of the cell lines compared to the erythrocytes should also be considered. The cell lines assessed have a diameter of 90-240 $\mu \mathrm{M}$, whereas the erythrocytes have a diameter of 6-8 $\mu \mathrm{M}[39,40]$. Therefore, it could be suggested that the erythrocytes are too small to attract the AMP, preventing haemolysis from occurring.

It is generally accepted that the selective cytotoxicity of AMPs towards bacteria and cancer cells is due to both cell types possessing a negative charge on their cell membranes. Therefore, we sought to investigate if antimicrobial activity could be used as an indicator of an AMPs anticancer potential. Bombinins $\mathrm{H} 2$ and $\mathrm{H} 4$ showed significant antimicrobial activity towards S. epidermidis 1457 and 5179-R1 at $100 \mu \mathrm{M}$, whilst temporins A and L exhibited some antimicrobial effects on the bacteria but not to the same extent as the bombinins. Given that bombinin $\mathrm{H} 2$ and temporin $\mathrm{L}$ were not selectively cytotoxic and temporin A demonstrated the most efficacious anticancer activity, the antimicrobial effect of an AMP cannot be used as an indicator for its anticancer potential. 


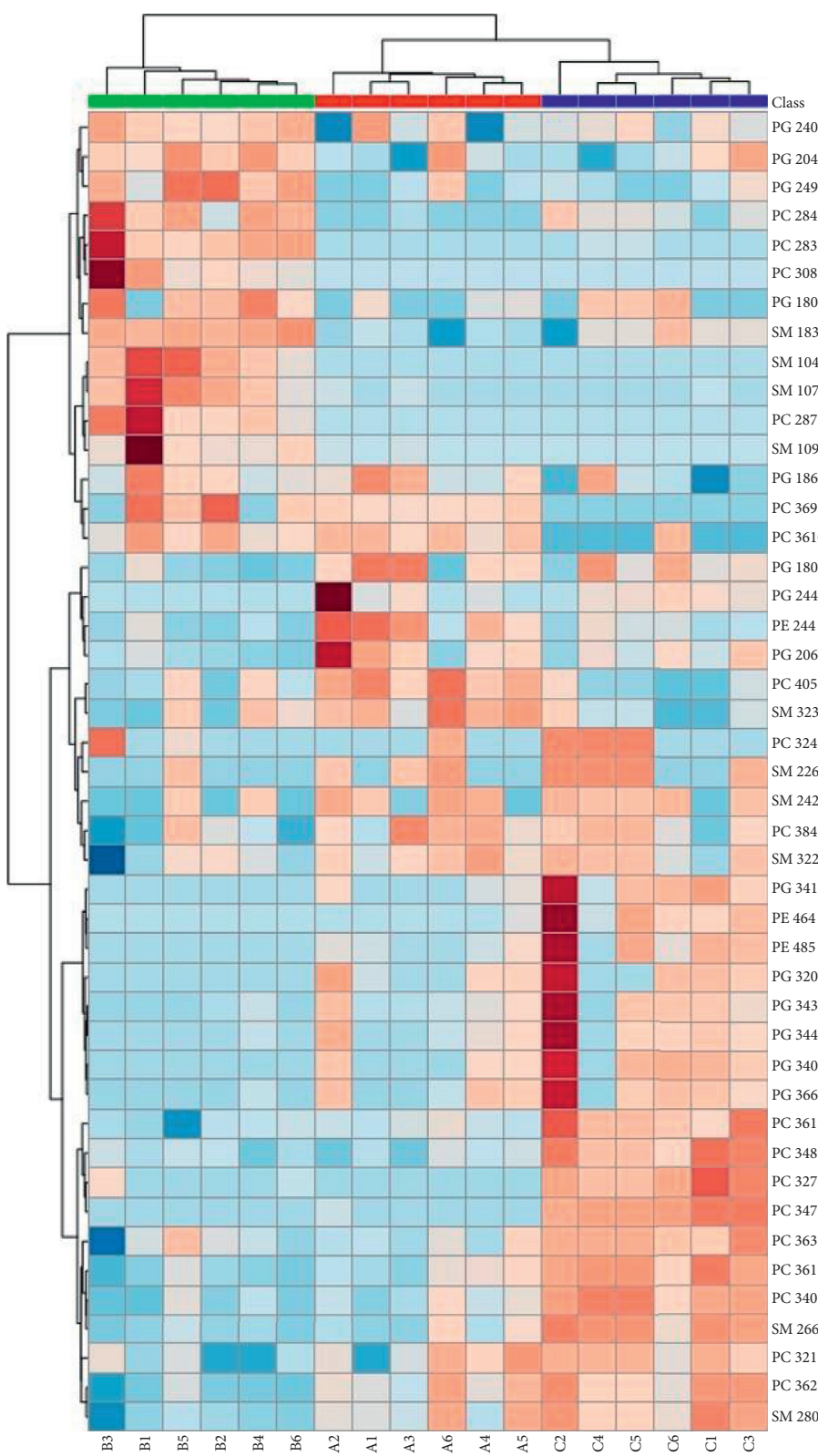

(a)

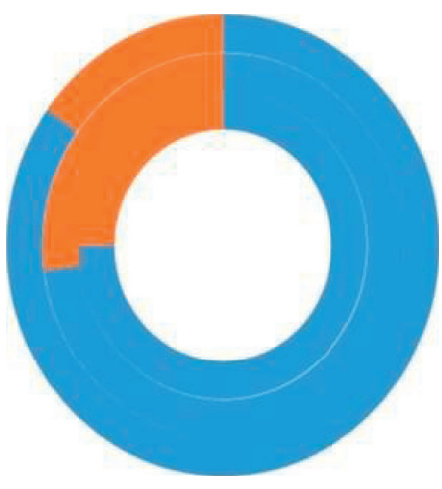

Positive

negative

FIgURE 4: (a) The relative intensity of the phospholipids showing differences between the cell lines, obtained via positive-mode MALDITOF. (b) The relative percentage of positive and negative phospholipids deemed as significantly different between the outer leaflets of the three cell lines.

\section{Conclusion}

Research on the anticancer properties of AMPs has increased in recent years. Many of these studies use different cancer cell lines but usually only one from a particular cancer type. Here we show that the effectiveness of AMPs as anticancer agents varies not just between AMPs but also between cancer types, even within a particular cancer type. Therefore, it is imperative that studies are conducted using more than one cell line from the same cancer type to ascertain if an AMP cytotoxicity is cell line specific. Furthermore, our preliminary investigation of four Anura AMPs demonstrated that temporin A and bombinin $\mathrm{H} 4$ may hold potential as anticancer agents, and further in vitro and in vivo work is now required to fully ascertain their anticancer potential and mode of action.

\section{Data Availability}

All data generated or analysed during the current study are included in this published article.

\section{Conflicts of Interest}

The authors declare that there are no conflicts of interest regarding the publication of this article. 


\section{Authors' Contributions}

LS carried out majority of the laboratory work and wrote the manuscript. CM was a major contributor to the writing of the manuscript as well as the main supervisor of the project. PC carried out membrane composition laboratory work. LGH provided technical support for the bacterial elements of the work. ED and KS provided technical support and data interpretation for the mass spectrometry work. PL was a secondary supervisor of the MRes project and helped with statistical analysis. FC provided the AMPs used and contributed to the review of the manuscript preparation. All authors read and approved the final manuscript.

\section{References}

[1] World Health Organisation (WHO), World Cancer Report 2018, International Agency for Research on Cancer, Lyon, France, 2018.

[2] J. H. Schiller, D. Harrington, C. P. Belani et al., "Comparison of four chemotherapy regimens for advanced non-small-cell lung cancer," New England Journal of Medicine, vol. 346, no. 2, pp. 92-98, 2002.

[3] Cancer Research UK, Cancer Statistics for the UK, Cancer Research UK, London, UK, 2016, http://www. cancerresearchuk.org/health-professional/cancerstatistics\#heading-Three.

[4] F. Fossella, J. R. Pereira, J. von Pawel et al., "Randomized, multinational, phase III study of docetaxel plus platinum combinations versus vinorelbine plus cisplatin for advanced non-small-cell lung cancer: the TAX 326 study group," Journal of Clinical Oncology, vol. 21, no. 16, pp. 3016-3024, 2003.

[5] H. Huang, J. Liu, Q. Meng, and G. Niu, "Multidrug resistance protein and topoisomerase 2 alpha expression in non-small cell lung cancer are related with brain metastasis postoperatively," International Journal of Clinical and Experimental Pathology, vol. 8, pp. 11537-11542, 2015.

[6] F. Pinheiro da Silva and M. C. C. Machado, "Antimicrobial peptides: clinical relevance and therapeutic implications," Peptides, vol. 36, no. 2, pp. 308-314, 2012.

[7] Y. Tian, H. Wang, B. Li et al., "The cathelicidin-BF Lys16 mutant Cbf-K16 selectively inhibits non-small cell lung cancer proliferation in vitro," Oncology Reports, vol. 30, no. 5, pp. 2502-2510, 2013.

[8] R. E. W. Hancock and G. Diamond, "The role of cationic antimicrobial peptides in innate host defences," Trends in Microbiology, vol. 8, no. 9, pp. 402-410, 2000.

[9] A. Izadpanah and R. L. Gallo, "Antimicrobial peptides," Journal of the American Academy of Dermatology, vol. 52, no. 3, pp. 381-390, 2005.

[10] D. W. Hoskin and A. Ramamoorthy, "Studies on anticancer activities of antimicrobial peptides," Biochimica et Biophysica Acta (BBA)-Biomembranes, vol. 1778, no. 2, pp. 357-375, 2008.

[11] R. Hancock and A. Patrzykat, "Clinical development of cationic antimicrobial peptides: from natural to novel antibiotics," Current Drug Target-Infectious Disorders, vol. 2, no. 1, pp. 79-83, 2002.

[12] D. Gaspar, A. S. Veiga, and M. A. Castanho, "From antimicrobial to anticancer peptides. A review," Frontiers in Microbiology, vol. 4, p. 294, 2013.
[13] C. Y. Huang, H. Y. Huang, M. D. Forrest, Y. R. Pan, W. J. Wu, and H. M. Chen, "Inhibition effect of a custom peptide on lung tumors," PLoS One, vol. 9, Article ID e109174, 2014.

[14] A. C. Rinaldi, "Antimicrobial peptides from amphibian skin: an expanding scenario: commentary," Current Opinion in Chemical Biology, vol. 6, no. 6, pp. 799-804, 2002.

[15] L. H. Lazarus and M. Attila, "The toad, ugly and venomous, wears yet a precious jewel in his skin," Progress in Neurobiology, vol. 41, no. 4, pp. 473-507, 1993.

[16] S. Charpentier, M. Amiche, J. Mester et al., "Structure, synthesis, and molecular cloning of dermaseptins B, a family of skin peptide antibiotics," Journal of Biological Chemistry, vol. 273, no. 24, pp. 14690-14697, 1998.

[17] Y. Ohsaki, A. F. Gazdar, H. C. Chen, and B. E. Johnson, "Antitumor activity of magainin analogues against human lung cancer cell lines," Cancer Research, vol. 52, no. 13, pp. 3534-8, 1992.

[18] J. Lehmann, M. Retz, S. S. Sidhu et al., "Antitumor activity of the antimicrobial peptide magainin II against bladder cancer cell lines," European Urology, vol. 50, no. 1, pp. 141-147, 2006.

[19] R. A. Cruciani, J. L. Barker, M. Zasloff, H. C. Chen, and O. Colamonici, "Antibiotic magainins exert cytolytic activity against transformed cell lines through channel formation," Proceedings of the National Academy of Sciences, vol. 88, no. 9, pp. 3792-3796, 1991.

[20] M. Simmaco, G. Mignogna, S. Canofeni, R. Miele, M. L. Mangoni, and D. Barra, "Temporins, antimicrobial peptides from the European red frog Rana temporaria," European Journal of Biochemistry, vol. 242, no. 3, pp. 788-792, 1996.

[21] G. Mignogna, M. Simmaco, G. Kreil, and D. Barra, "Antibacterial and haemolytic peptides containing D-alloisoleucine from the skin of Bombina variegata," The EMBO Journal, vol. 12, no. 12, pp. 4829-4832, 1993.

[22] J. M. Conlon, M. Mechkarska, M. L. Lukic, and P. R. Flatt, "Potential therapeutic applications of multifunctional hostdefense peptides from frog skin as anti-cancer, anti-viral, immunomodulatory, and anti-diabetic agents," Peptides, vol. 57, pp. 67-77, 2014.

[23] K. C. Mulder, L. A. Lima, V. J. Miranda, S. C. Dias, and O. L. Franco, "Current scenario of peptide-based drugs: the key roles of cationic antitumor and antiviral peptides," Front Microbiol, vol. 4, p. 321, 2013

[24] R. Estrada and M. C. Yappert, "Alternative approaches for the detection of various phospholipid classes by matrix-assisted laser desorption/ionization time-of-flight mass spectrometry," Journal of Mass Spectrometry, vol. 39, no. 4, pp. 412-422, 2004.

[25] V. Luca, D. Barra, and M. Mangoni, Handbook of Biologically Active Peptides, Elsevier, Amsterdam, Netherlands, Second edition, 2013.

[26] L. J. Swithenbank, The Effects of Antimicrobial Peptides from Lucilia sericata and Anurans on Non-small Cell Lung Cancer, Masters thesis, Swansea University, Swansea, UK, 2017.

[27] E. Bevers, P. Comfurius, and R. Zwaal, "Regulatory mechanisms in maintenance and modulation of transmembrane lipid asymmetry: pathophysiological implications," Lupus, vol. 5, no. 5, pp. 480-487, 1996.

[28] S. Riedl, B. Rinner, H. Schaider, K. Lohner, and D. Zweytick, "Killing of melanoma cells and their metastases by human lactoferricin derivatives requires interaction with the cancer marker phosphatidylserine," BioMetals, vol. 27, no. 5, pp. 981-997, 2014. 
[29] S. Beloribi-Djefaflia, S. Vasseur, and F. Guillaumond, "Lipid metabolic reprogramming in cancer cells," Oncogenesis, vol. 5, no. 1, p. e189, 2016.

[30] B. Chen, W. Le, Y. Wang et al., "Targeting negative surface charges of cancer cells by multifunctional nanoprobes," Theranostics, vol. 6, no. 11, pp. 1887-1898, 2016.

[31] Y. Pouny, D. Rapaport, A. Mor, P. Nicolas, and Y. Shai, "Interaction of antimicrobial dermaseptin and its fluorescently labeled analogs with phospholipid membranes," Biochemistry, vol. 31, no. 49, pp. 12416-12423, 1992.

[32] M. Dathe, M. Schümann, T. Wieprecht et al., "Peptide helicity and membrane surface charge modulate the balance of electrostatic and hydrophobic interactions with lipid bilayers and biological membranes," Biochemistry, vol. 35, no. 38, pp. 12612-12622, 1996.

[33] Y. Shai and Z. Oren, "Diastereomers of cytolysins, a novel class of potent antibacterial peptides," Journal of Biological Chemistry, vol. 271, no. 13, pp. 7305-7308, 1996.

[34] D. Avrahami, Z. Oren, and Y. Shai, "Effect of multiple aliphatic amino acids substitutions on the structure, function, and mode of action of diastereomeric membrane active peptides," Biochemistry, vol. 40, no. 42, pp. 12591-12603, 2001.

[35] A. Hawrani, R. A. Howe, T. R. Walsh, and C. E. Dempsey, "Origin of low mammalian cell toxicity in a class of highly active antimicrobial amphipathic helical peptides," Journal of Biological Chemistry, vol. 283, no. 27, pp. 18636-18645, 2008.

[36] C. Oelkrug, M. Hartke, and A. Schubert, "Mode of action of anticancer peptides (ACPs) from amphibian origin," Anticancer Research, vol. 35, no. 2, pp. 635-643, 2015.

[37] D. Raucher and J. S. Ryu, "Cell-penetrating peptides: strategies for anticancer treatment," Trends in Molecular Medicine, vol. 21, no. 9, pp. 560-570, 2015.

[38] J. D. Clogston and A. K. Patri, "Zeta potential measurement," Methods in Molecular Biology, vol. 697, pp. 63-70, 2011.

[39] G. L. Castoldi and L. Del Senno, "Erythrocytes," Encyclopedia of Immunologypp. 833-841, Elsevier, Amsterdam, Netherlands, 2nd edition, 1998.

[40] X. Li, S. Yan, J. Dai et al., "Human lung epithelial cells A549 epithelial-mesenchymal transition induced by PVA/Collagen nanofiber," Colloids and Surfaces B: Biointerfaces, vol. 162, pp. 390-397, 2018. 